\title{
The Ascending Aorta Pseudoaneurysm with Myocardium Rupture Complicated with Prosthetic Valve Infective Endocarditis after Aortic Valve Replacement
}

Atsushi Kobayashi ${ }^{*}$, Kazuhiko Nakazato, Yuichiro Jin, Hiroyuki Yamauchi, Takashi Kaneshiro, Masayoshi Oikawa, Hitoshi Suzuki, Shu-ichi Saitoh and Yasuchika Takeishi

Department of Cardiology and Hematology, Fukushima Medical University, Japan

*Corresponding author: Atsushi Kobayashi, MD, Department of Cardiology and Hematology, Fukushima Medical University, Fukushima, Japan 960-1295, Fax: +81-24-548-1821, Tel: +81-24-547-1190; E-mail: koba-a@fmu.ac.jp

Received date: Mar 17, 2014, Accepted date: Apr 9, 2014, Published date: Apr 16, 2014

Copyright: (c) 2014 Kobayashi A, et al. This is an open-access article distributed under the terms of the Creative Commons Attribution License, which permits unrestricted use, distribution, and reproduction in any medium, provided the original author and source are credited.

Abbreviations

AVR: Aortic Valve Replacement; CAVB: Complete Atrioventricular Block; PVE: Prosthetic Valve Infective Endocarditis

A 41-year-old man was referred to our hospital complaining of epigastralgia and back pain lasting for 1 month and had consistently elevated plasma CRP levels. His past medical history included aortic valve replacement (AVR) due to aortic regurgitation at the age of 39 years and pacemaker implantation because of complete atrioventricular block (CAVB) 6 months before the present admission. Transthoracic echocardiography demonstrated a pseudoaneurysm of the nearby left ventricular septum and rupture of the left ventricular myocardium by enlargement of the pseudoaneurysm (Figures 1 and 2).

The pseudoaneurysm, which invaded the left ventricular myocardium, dilated during diastole, but no connection to the left ventricular cavity was observed. Transthoracic echocardiography also showed reduced motion of the inferior left ventricular wall. The prosthetic valve was not destabilized, and prosthetic valvular function was normal. Furthermore, transesophageal echocardiography demonstrated the pseudoaneurysm to be in the posterior aortic root (Figure 3).

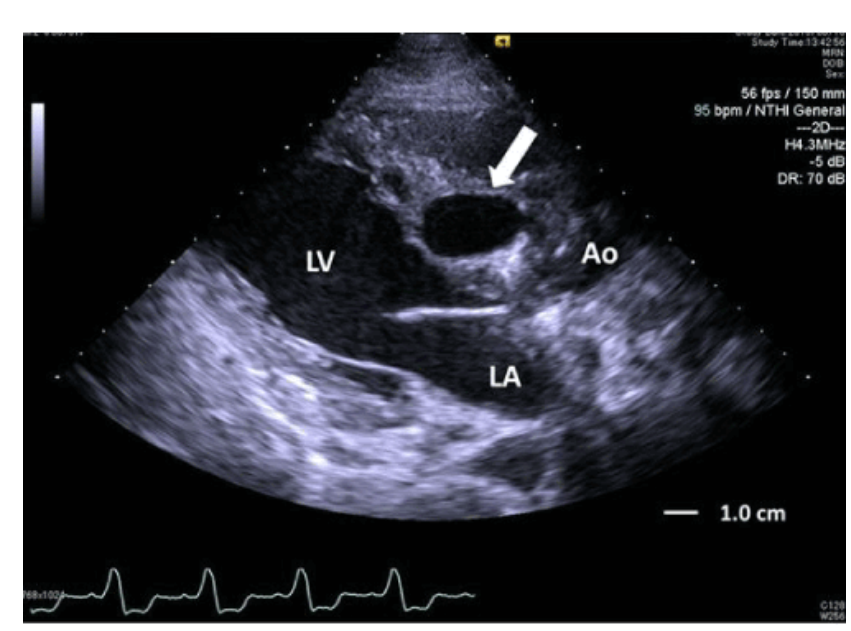

Figure 1: Transthoracic echocardiography. Parasternal long axis view demonstrating a pseudoaneurysm (arrow). LV indicates left ventricle; LA, left atrium; Ao, aorta.

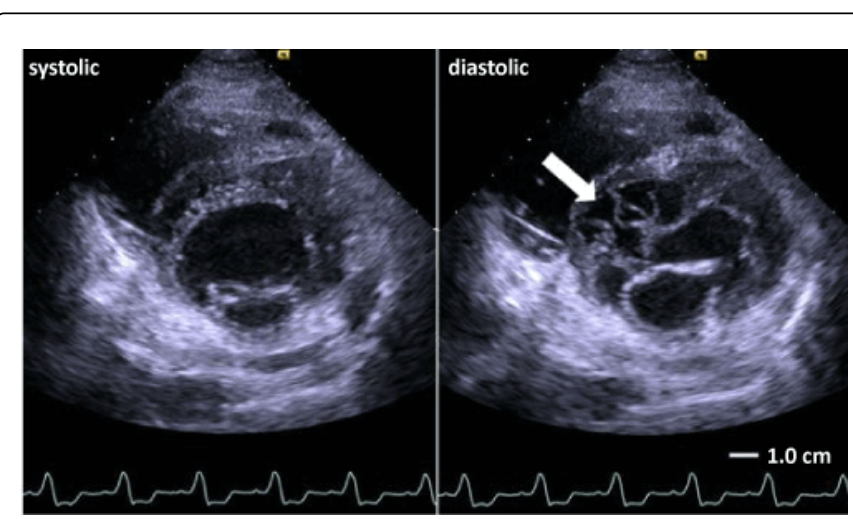

Figure 2: Transthoracic echocardiography Parasternal short axis view demonstrating the pseudoaneurysm (arrow) into the left ventricular myocardium.

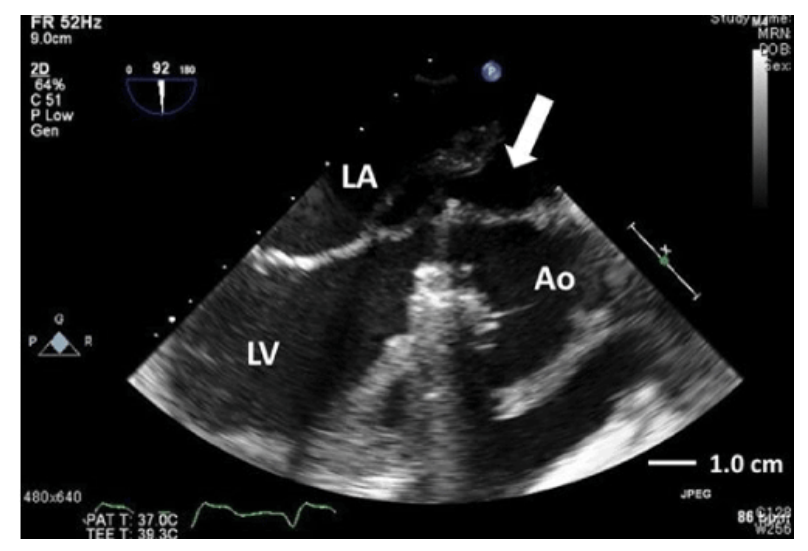

Figure 3: Transesophageal echocardiography demonstrating the pseudoaneurysm (arrow) to be in the posterior aortic root.

A cardiac 3-dimensional computed tomography scan showed that the pseudoaneurysm was widespread around aortic root ring and into the left ventricular myocardium (Figure 4). 
Page 2 of 2

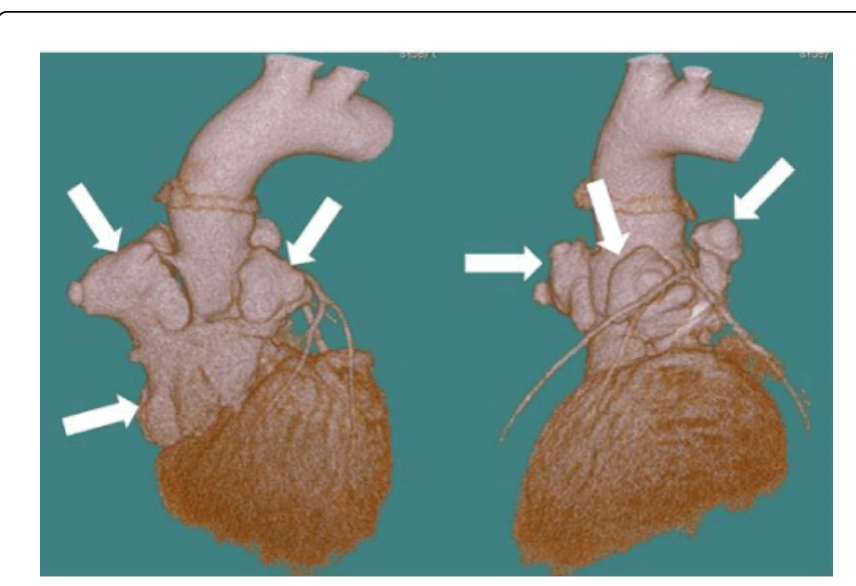

Figure 4: A cardiac 3-dimensional computed tomography scan demonstrated that the pseudoaneurysm (arrows) was widespread around aortic root ring and into the left ventricular myocardium.

The scan also showed a compression of the proximal right coronary artery by the pseudoaneurysm (Figure 4). The patient underwent surgical treatment, and a perivalvular abscess was found. Pseudoaneurysm of the ascending aorta after AVR is a rare and dreadful complication, which may occur several months or years after aortic surgery. In the present case specifically, the ascending aorta pseudoaneurysm was widespread into the left ventricular myocardium. The mechanism of pseudoaneurysm after AVR is unclear. Defects in the aortotomy suture lines, fragility of the aorta, extensive calcification in the aortic wall, iatrogenic trauma due to manipulation during AVR, and infection are some of the possibilities $[1,2]$.
A perivalvular abscess was found 2 years after AVR in the present case. The patient had a history of pacemaker implantation 6 months previously, due to CAVB. CAVB might be is a complication of the perivalvular abscess. Diagnosis of prosthetic valve infective endocarditis (PVE) is challenging and difficult [3].

The low survival rate for this disease is related to deficiencies of identifying prosthetic and periprosthetic damage via echocardiography, which can lead to a delay in the application of antibiotic and surgical therapies. A recent study has indicated that the use of positron emission tomography with $18 \mathrm{~F}$-fluorodeoxyglucose is helpful for diagnosing PVE [4].

We suggest early detection and early intervention of PVE and pseudoaneurysm after AVR as they are fatal complications.

\section{References}

1. Bachet J, Pirotte M, Laborde F, Guilmet D (2007) Reoperation for giant false aneurysm of the thoracic aorta: how to reenter the chest? Ann Thorac Surg 83: 1610-1614.

2. Bonnichsen CR, Sundt TM III, Anavekar NS, Foley TA, Morris MF (2011) Aneurysms of the ascending aorta and arch: the role of imaging in diagnosis and surgical management. Expert Rev Cardiovasc Ther 9: 45-61.

3. Habib G, Thuny F, Avierinos JF (2008) Prosthetic valve endocarditis: current approach and therapeutic options. Prog Cardiovasc Dis 50: 274281.

4. Saby L, Laas O, Habib G, Cammilleri S, Mancini J, et al. (2013) Positron emission tomography/computed tomography for diagnosis of prosthetic valve endocarditis: increased valvular (18)F-fluorodeoxyglucose uptake as a novel major criterion. J Am Coll Cardiol 61: 2374-2382.

\footnotetext{
This article was originally published in a special issue, entitled: "Cardiovascular Diseases and Case Reports", Edited by Wilbert S. Aronow, New York Medical College, USA
} 